\title{
RESULTS OF THE PISCES-PERSEUS HI REDSHIFT SURVEY
}

\author{
Riccardo Giovanelli ${ }^{1}$ and Martha P. Haynes ${ }^{2}$ \\ NAIC $^{3}$, Arecibo Observatory and Astronomy Dept., Cornell University \\ USA
}

\begin{abstract}
We report on the current status of the HI redshift survey in the Pisces-Perseus supercluster and present results based on a sample of approximately 4700 redshifts known in the $6^{h}$ by $45^{\circ}$ region of the sky dominated by the supercluster. In particular we derive density contrasts between voids and high density regions and discuss the existence of a luminosity bias between galaxies that reside in regions of different local density. Such a bias is not the consequence of the well-known pattern of morphological type segregation, but results from a difference in the shape of the luminosity function of galaxies in high and low density regions. Bright galaxies tend to be relatively less abundant, and faint ones relatively more so, in low density environments.
\end{abstract}

\section{CURRENT STATUS OF THE SURVEY}

For several years, we have devoted a substantial effort to the observation of several thousand galaxies in the general region of the local universe dominated by the Pisces-Perseus supercluster. The goals of the survey and partial results are described in Giovanelli and Haynes (1985) and Giovanelli et al. (1986). The survey aims toward completion to the magnitude limit of the CGCG (Zwicky et al. 1961-68), $m=15.7$, and to the angular size limit of the UGC (Nilson 1973), $a=1.0^{\prime}$ within the region roughly bound by R.A. $=\left[22^{h}, 04^{h}\right]$ and $\mathrm{Dec}=\left[0^{\circ},+45^{\circ}\right]$. Morphological classification and size measurement of all non-UGC galaxies has been performed using glass copies of the blue Palomar Sky Survey, supplemented in some areas by higher quality plate material. Three-quarters of the catalogued galaxies in the region are spirals, and constitute good targets for an HI redshift survey at Arecibo. $21 \mathrm{~cm}$ data for about 3000 objects have been supplemented with measurements from various other sources, to comprise a redshift sample totaling 4700 objects in the southern galactic cap north of the celesial equator. The completion percentages vary somewhat over the region, being lower in the extreme North and South parts, due to reduced sensitivity at high zenith angles of the current feed system at the $305 \mathrm{~m}$ telescope and the lower sensitivity of the NRAO ${ }^{4}$. They are also mildly dependent on morphological type as the HI effort can only be directed to galaxies of type $\mathrm{SO}$ a or later; extension of the survey to lenticular and elliptical galaxies depends on optical studies. Fortunately, the morphological segregation that is responsible for the higher tendency towards clustering for early type galaxies,- which also tend to be of higher optical surface brightness - has also made them favorite targets of optical observers. Thus the integration of existing optical and HI data yields an appreciably well-matched overall sample. Complementary optical

1 Arecibo Observatory, P.O. Box 995, Arecibo P.R. 00613, USA

2 Astronomy Dept., Space Sciences Building, Cornell University, Ithaca, NY 14853, USA

3 The National Astronomy and Ionosphere Center is operated by Cornell University under cooperative agreement with the National Science Foundation.

4 The National Radio Astronomy Observatory is operated by Associated Universities, Inc. under contract with the National Science Foundation. 


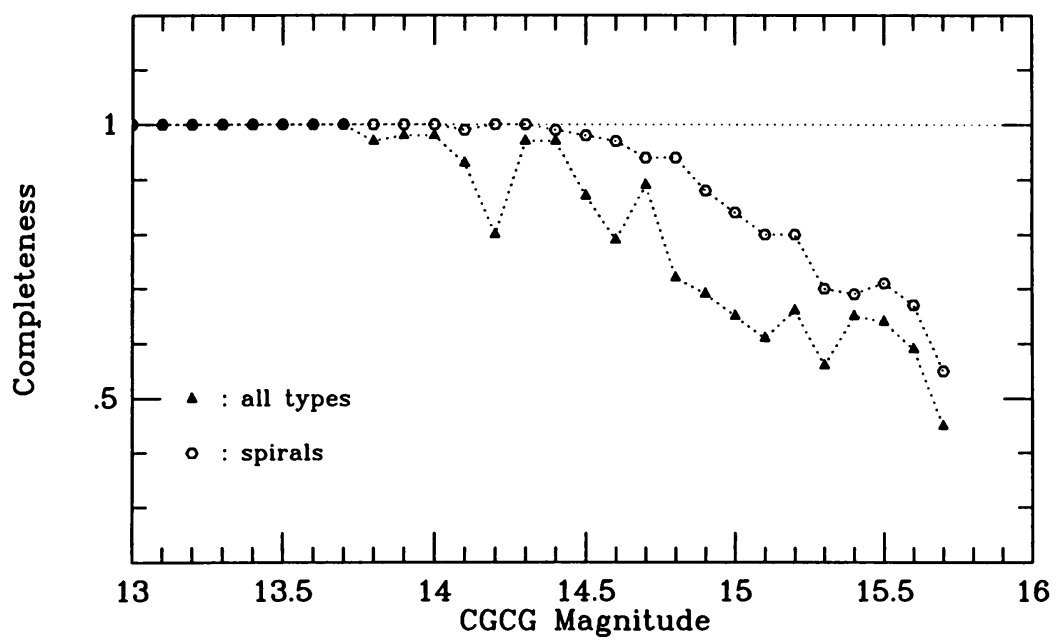

Figure 1. Completeness fraction in the region $\mathrm{RA}=\left[21.5^{h}, 04^{h}\right] \mathrm{Dec}=\left[21.5^{\circ}, 33.5^{\circ}\right]$.

observations and efforts by other groups are helping to reach completion of the early-type population as well.

Figure 1 illustrates the degree of completion obtained in the strip between Declinations of $21^{\circ} 30^{\prime}$ and $33^{\circ} 30^{\prime}$. In this region, 1460 redshifts are known; $70 \%$ were obtained at $21 \mathrm{~cm}$. This region best illustrates the shortcomings of the survey. In the figure, completion is defined as the ratio between the number of galaxies of known redshift to that of all CGCG galaxies in a given $0.1 \mathrm{mag}$ bin. Completion is plotted separately for all types and for spiral galaxies alone. The percentages of the redshift sample in this region are $6 \% \mathrm{E}$ 's, $11 \% \mathrm{SO}$ 's and $83 \%$ Spirals, marginally more spiral-rich than the canonical 10-10-80 population ratio found in the general field. When all types are included, completion is better than 50 percent for all bins except for the one at $\mathrm{m}=15.7$. For the overall sample at all declinations, completion is better than $50 \%$ at $\mathrm{m} \leq 15.5$. It should be further added that for $21 \mathrm{~cm}$ non-detections, we typically have a reliable estimate of the velocity range in which the galaxy is likely not to be found, i.e. the $21 \mathrm{~cm}$ search window, usually $\mathrm{v}_{0}<15000$ $\mathrm{km} \mathrm{s}^{-1}$.

\section{VOLUME DENSITIES AND DENSITY CONTRASTS}

We have used the redshift sample to obtain an absolute magnitude limited subsample very nearly complete to a distance of $7500 \mathrm{~km} \mathrm{~s}^{-1}$ and $\mathrm{M}=-19.5$ over the solid angle of the survey. This subsample includes only about $1 / 5$ of the total, but with minor corrections for incompleteness it allows us to obtain a true 3-dimensional volume density map of the distribution of bright galaxies. The gridding of the volume is cartesian, with cells of $200 \mathrm{~km} \mathrm{~s}^{-1}$ to the side and the origin at the Earth, with $x$-axis toward $(\mathrm{RA}, \mathrm{Dec})=\left(22^{h}, 0^{\circ}\right), y$-axis toward $(\mathrm{RA}, \mathrm{Dec})=\left(04^{h}, 0^{\circ}\right)$ and $z$-axis toward Dec $=90^{\circ}$. The outer boundaries are the Celestial Equator, a sphere of radius of 7500 $\mathrm{km} \mathrm{s}^{-1}$ and the cone of the Dec $=45^{\circ}$ surface. Fig. 2 displays two slices taken perpendicular to the $z$-axis, where the intersections of each slice with the two latter bounding surfaces are outlined by dashed lines; the height above the Celestial Equator is respectively 600 and $2400 \mathrm{~km} \mathrm{~s}^{-1}$, as indicated in each panel. The slices are "integrated" in the $z$ direction over one cell length, i.e. 200 $\mathrm{km} \mathrm{s}^{-1}$. They illustrate the type of structures encountered in this region without the "polar" bias 
of Earth-centered cone diagrams, and the density array that they slice provides a useful tool to compare with the analogous numerical simulations. The $\mathrm{V}_{z}$ $=600 \mathrm{~km} \mathrm{~s}^{-1}$ slice displays a "void" centered near $3000 \mathrm{~km} \mathrm{~s}^{-1}$ surrounded by low density enhancements and a more conspicuous, but not fully outlined one beyond $6000 \mathrm{~km} \mathrm{~s}^{-1}$ which extends outside the boundary of the volume. The $\mathrm{V}_{z}=2400 \mathrm{~km} \mathrm{~s}^{-1}$ slice exhibits a cut through the "filament" that represents the main continuous density enhancement of the region and does not include the Perseus cluster, which is at a somewhat higher $\mathrm{V}_{z}$. These two structures well illustrate the types of large scale structures that are more easily detectable in the distribution of galaxies in this region. More conventional displays of the data are given in Giovanelli, Haynes and Chincarini (1986) and Haynes and Giovanelli (1986).

With the density array $n(x, y, z)$, the outlines of the filament can be traced by following equidensity surfaces. With some subjective truncation at branching points, we obtain the outline of a solid bound by a single surface, roughly fit by a very prolate spheroid with a major to minor axial ratio of about 10 and inclined by less than $12^{\circ}$ to the line of sight. Rather than to provide a strict topological description [studies of that nature are currently being carried in collaboration with Bhavsar, Chincarini, Gott, Melott and Weinberg] this exercise is meant to obtain a useful tool for measuring density contrasts. It is worth noting however that, while structures of the type of the Pisces-Perseus filament do not seem to be common in the local universe, other examples of strongly elongated geometry exist (e.g. the Lynx-Ursa Major supercluster, Giovanelli and Haynes 1982).

The top curve in Fig. 3 displays the contrast between the actual number of galaxies counted within a given redshift shell and that which would be expected from a uniform density distribution, within the solid angle subtended by the density enhancement represented by the filament. Because the filament is slightly inclined to the line of sight, smearing of the density contrast in velocity results; nonetheless, the filament represents a density enhancement that averages in excess of a factor 10 greater than the mean density of galaxies. A correction for the inclination to the line of sight would bring that number closer to 20 . A slight underdensity is present in the foreground, a reminder of the existence of a nearby void.
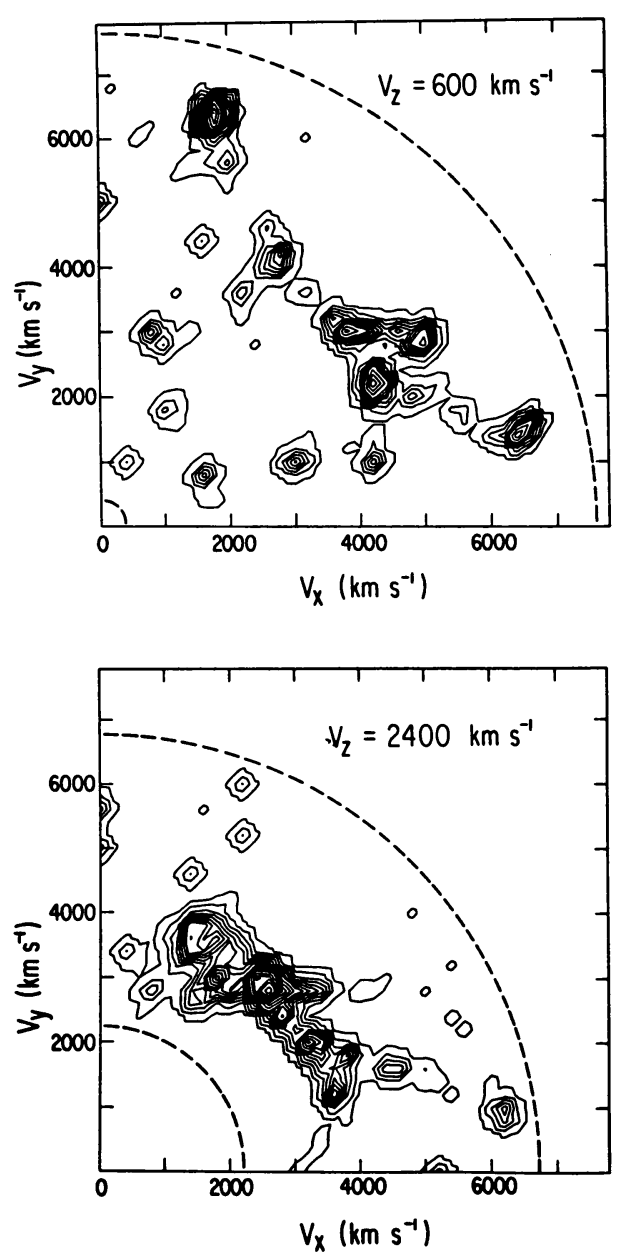

Fig. 2. Two slices of the density array $n(x, y, z)$. Both slices are parallel to the $\left(V_{x}, V_{y}\right)$ plane, i.e. the celestial equator. Contours are of equal density of galaxies brighter than $M=-19.5$. The outer dashed circular curve is the intersection of the slice with the sphere of radial velocity $7500 \mathrm{~km}$ $\mathrm{s}^{-1}$ while the inner one is the intersection with the cone given by $\mathrm{Dec}=45^{\circ}$. 


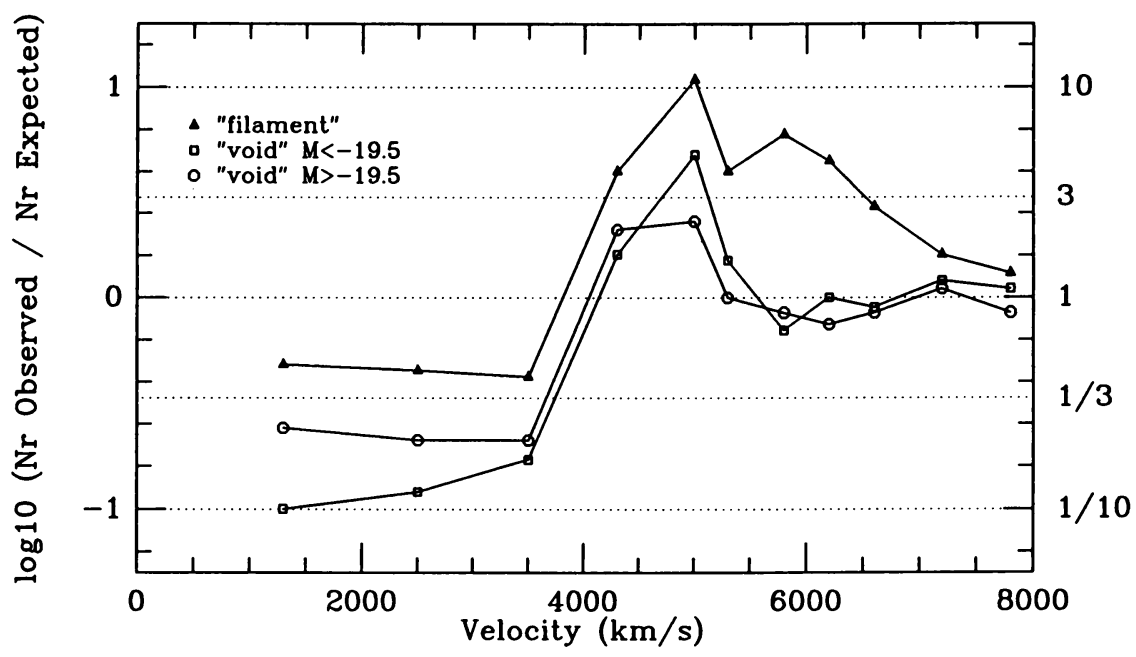

Figure 3. Density contrast profiles : the "filament" line refers to the solid angle subtended by the high density region in the Pisces-Perseus supercluster, the other two to the solid angle subtended by a foreground void. Each point corresponds to the ratio between observed and expected galaxy counts within a redshift shell centered on that point's abscissa. The two "void" curves refer to galaxies in different absolute magnitude bins, as indicated.

Similarly to what we did for the filament, from the density array we can obtain an outline of the solid angle subtended by the nearby void briefly discussed in connection with Fig. 2. Then we can compare galaxy counts within the solid angle subtended by the void and compare them, in contrast, with those expected from a uniform distribution, separately for bright $(\mathrm{M}<-19.5)$ and faint $(M>-19.5$, to the catalog limit within the given shell) galaxies. The resultant profiles are illustrated in the two bottom curves in Fig. 3. The partial overlap of solid angles subtended by the void and the filament is responsible for the overdensity near $5000 \mathrm{~km} \mathrm{~s}^{-1}$. We note here that (a) the mean underdensity of the void is at least a factor 5 and (b) the density contrast of the void is better outlined by the bright than by the faint galaxies.

We partially conclude that large-scale (i.e. on scales larger than cluster size) density contrasts between high and low density regions as high as a factor of 100 are observed, and that such a contrast appears to be dependent on the luminosity level of the galaxies counted.

\section{LUMINOSITY SEGREGATION}

\subsection{Luminosity Functions}

The distribution of luminous matter is highly inhomogeneous. As seen in the preceding Section, superclusters and voids yield large-scale deviations from the mean density by factors on the order of ten. Some currently popular theories require that the overall mass distribution be significantly smoother, thus leading to the postulate that the formation of luminous galaxies is a biased process (see Dekel and Rees 1987 for a recent review). This bias would translate to a spatial segregation of the low luminosity, low surface brightness galaxies from the bright, massive ones. An extreme corollary of this proposition is that low luminosity galaxies may fill the voids, which themselves are 
defined only by the brightest objects.

In order to test the bias hypothesis, we can derive luminosity functions (LF's) separately for high and low density regions using our redshift sample and $n(x, y, z)$ arrays of the type defined in Section 2. In order to best utilize the data sample and to avoid a Malmquist bias (which would arise if high and low density regions were not well mixed in the sampled volume, i.e. if the median distance of cells of low $n$ and that of those of high $n$ were not the same), we separately compare LF's in different redshift intervals, within each of which high and low $n$ cells are well mixed. For each interval, we compare the LF's of galaxies found in high and low density cells. Here, we derive density arrays in two velocity intervals, that for $v<4000 \mathrm{~km} \mathrm{~s}^{-1}, n_{1}(x, y, z)$, and that for $4000 \leq v \leq 6200$ $\mathrm{km} \mathrm{s}^{-1}, n_{2}(x, y, z)$. The first is obtained from a subsample with an absolute magnitude limit of -18.4 , while in the second the limit is -19.0 . Given the difference in luminosity depth, counts in $n_{1}$ for a standard Schechter LF should be about twice as large as those for $n_{2}$.

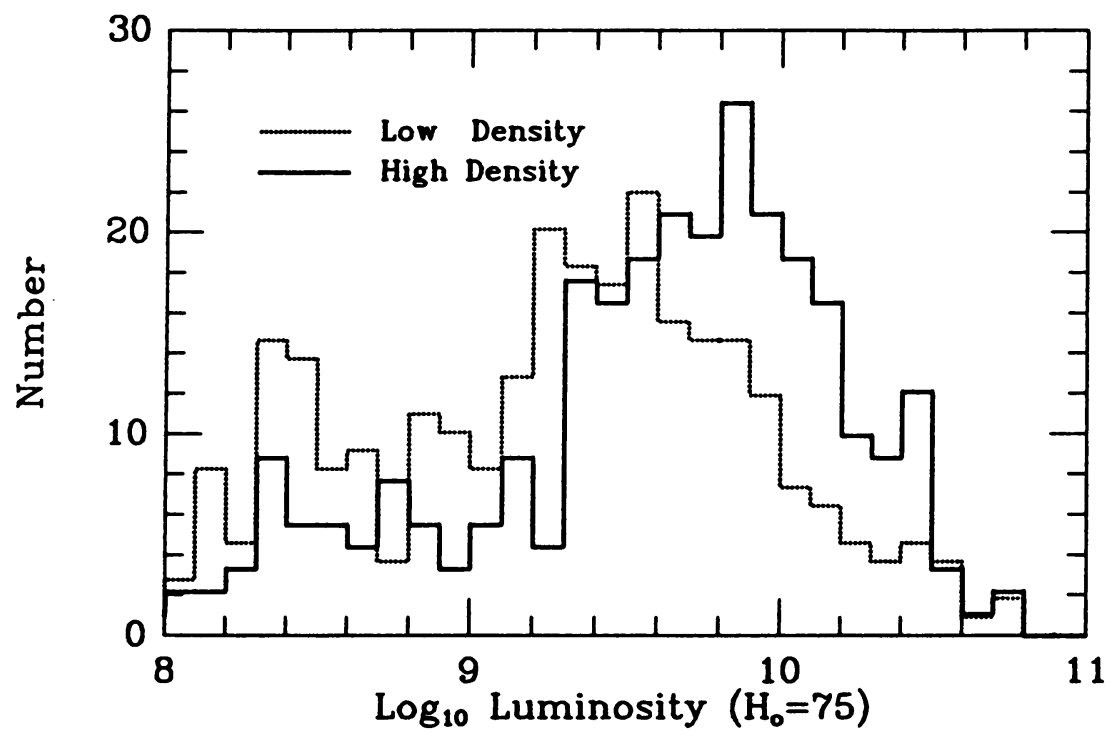

Figure 4. Photographic luminosity, in solar units, in the interval $0-4000 \mathrm{~km} \mathrm{~s}^{-1}$, separately for galaxies located in high and low density cells. Space densities derive from a subsample nearly complete to $M=-18.4$, which yields $\left\langle\log _{10} n_{1}\right\rangle=-2.2$, with $n_{1}$ in $\mathrm{Mpc}^{-3}$. The "high density" LF was obtained from galaxies located in cells with $\log _{10} n_{1} \geq-2.0$, the "low density" LF from those in cells with $\log _{10} n_{1}<-2.1$.

Fig. 4 displays LF's for the $v<4000 \mathrm{~km} \mathrm{~s}^{-1}$ interval, while Fig. 5 displays those for the higher velocity interval. In Fig. 4, the "high" and "low" density subsamples refer to the approximately 270 galaxies in the cells with highest and lowest $n_{1}$; similarly for Fig.5, although subsample size in this case numbers about 300 . However, the low velocity interval contains few galaxies in truly high density regimes, and the galaxies that make up the "high" density LF in Fig.4 are more similar in their density distribution to the "low" density subsample in Fig. 5 than to the "high" density subsample. The mean density in the $n_{1}$ and $n_{2}$ arrays are respectively 6 and $4.7 \times 10^{-3}\left(H_{0} / 75\right)$ galaxies $\mathrm{Mpc}^{-3}$. The fact that $\left.<n_{1}\right\rangle$ is smaller than $2 \times<n_{2}>$ is in part due to the presence of the nearby void. In Fig. 4, low and high density regimes are defined by the delimiters $\log _{10} n_{1}<-2.1$ and $\log _{10} n_{1}>-2.0$, while in Fig. 5 the corresponding delimiters are $\log _{10} n_{2}<-2.1$ (with most cells very close to this limit) and $\log _{10} n_{2}>-1.5$. The nuisance of having two different density scales 


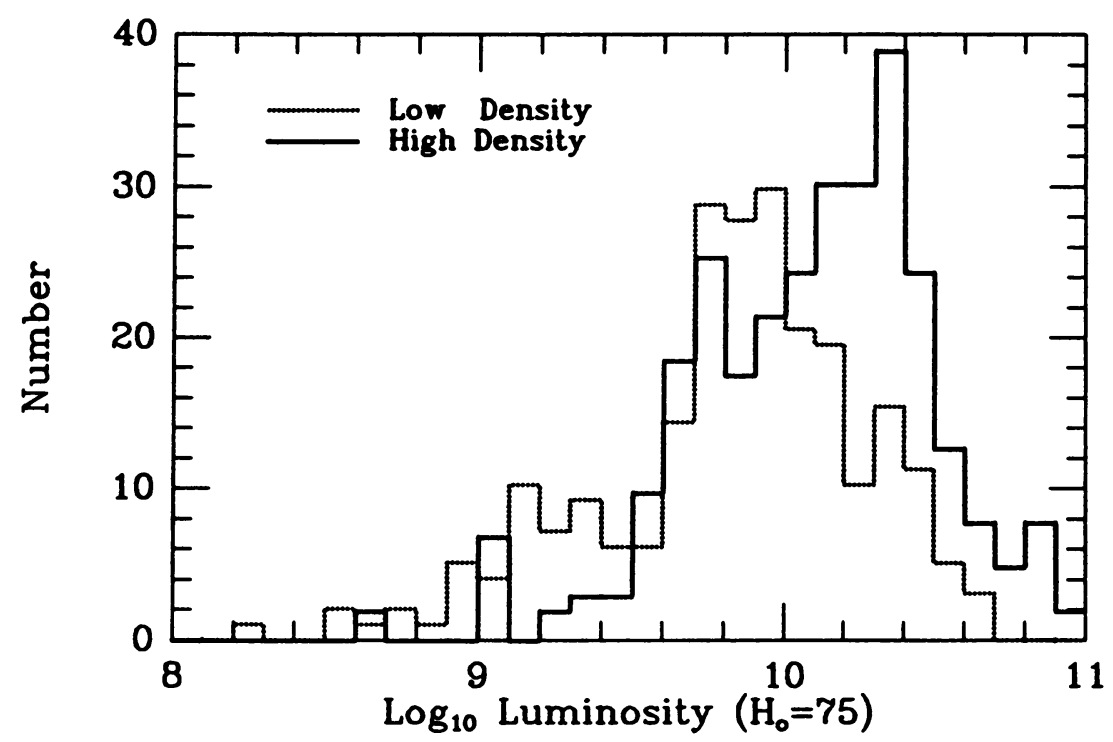

Figure 5. Photographic luminosity of galaxies in the interval $4000-6200 \mathrm{~km} \mathrm{~s}^{-1}$. Space densities derive from a subsample nearly complete to $M=-19.0$, which yields $<\log _{10} n_{2}>=-2.3$, with $n_{2}$ in galaxies, brighter than -19.0 , per Mpc ${ }^{3}$. The high density LF was obtained from galaxies located in cells with $\log _{10} n_{2}>-1.5$, while the low density one from galaxies in cells with $\log _{10} n_{2}<-2.1$. Reasonable completeness is available here for galaxies brighter than $10^{10} L_{\odot}$.

is the price paid to obtain better statistics. In both figures, the galaxies fainter than the limits of near completeness have been included; those limits in luminosity units are $10^{9.7} L_{\odot}$ and $10^{10.0} L_{\odot}$ for Figs. 4 and 5 respectively. Luminosities are corrected for galactic and internal extinction as described in Haynes and Giovanelli (1984). In both velocity regimes, there are relatively fewer high luminosity galaxies in the low density regions. And with the caution required by the incompleteness, the opposite appears to be true for low luminosity galaxies. Restriction of the samples to mid-spirals alone, yields an analogous pattern.

\subsection{Surface Magnitude}

As done for luminosity, surface magnitude distributions of galaxies in high and low density regimes are displayed in Fig. 6. "High" and "low" densities are defined by the delimiters $\log _{10} n_{2}<$ -2.1 and $\log _{10} n_{2}>-1.5$, and all galaxies within $6200 \mathrm{~km} \mathrm{~s}^{-1}$ are included. Surface magnitude is defined as the magnitude of the galaxy, corrected for extinction and galactic absorption, divided by the square of the major angular diameter of the blue image, as measured on the PSS, in units of mag $\operatorname{arcsec}^{-2}$. The effects of Malmquist bias, while not negligible, are less marked than for luminosity. Break down by velocity range reveals the same features visible in Fig. 6: galaxies in high density regions, which tend to be brighter, also have higher surface magnitude.

\subsection{Discussion}




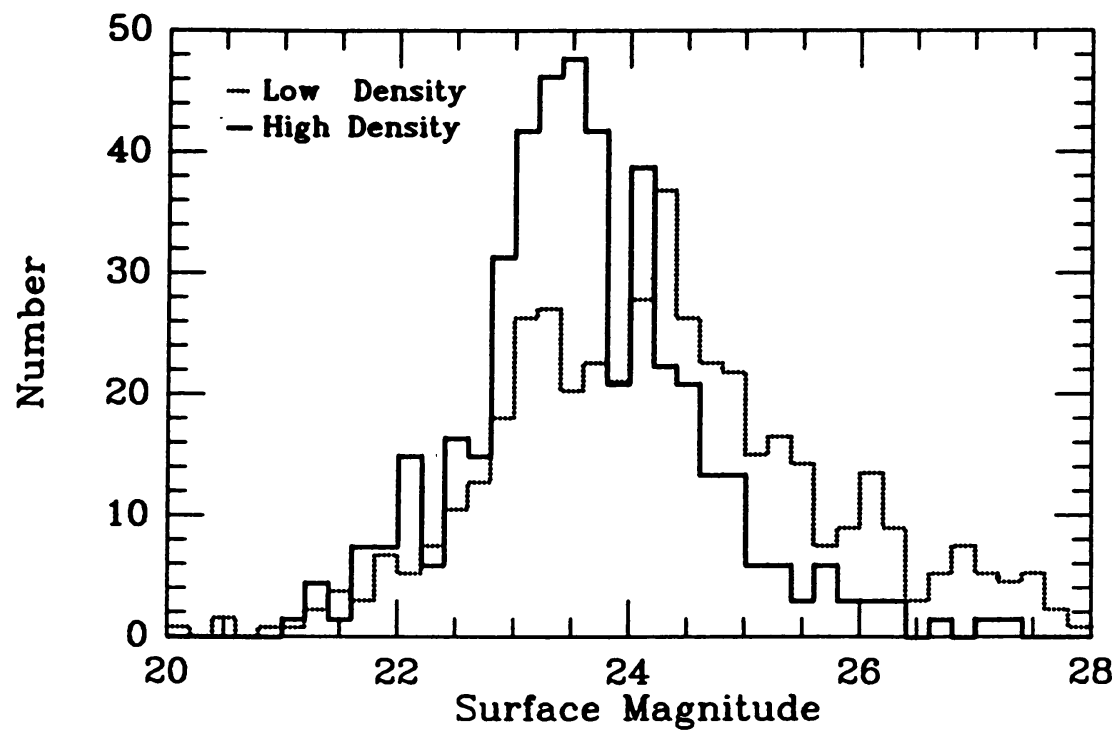

Figure 6. Histograms of surface magnitude, in mag $\operatorname{arcsec}^{-2}$.

It may be argued that in part, the effect seen in Figs. 4-6 is a consequence of morphological segregation, as follows. The clustering properties of galaxies varies monotonically with morphological type (Giovanelli, Haynes and Chincarini 1986) and with surface brightness (Davis and Djorgovski 1985). Because the LF differs for galaxies of different type (Sandage, Binggeli and Tammann 1985:SBT), different mixtures of types may yield different total LF's. We have generated "synthetic" LF's by starting from SBT's single type LF's and varying the relative type fraction, and found that the shape of the total LF is very resilient, for any sensible combination of types. A change in the mean surface magnitude between high and low density regions is easier to understand, as E's and S0's are notoriously of higher surface magnitude than late spirals and irregulars, and these two extremes in the morphological scale are well segregated by density regime. A surface magnitude segregation has also been found by Davis and Djorgovski (1985).

These results are however at odds with those obtained by Bothun etal.(1986) and Oemler (1987). Those authors found that the outlines of voids in redshift histograms of galaxies within the solid angles subtended by the voids themselves, appear substantially the same whether redshifts of high or low luminosity (or surface brightness) galaxies are plotted. In Giovanelli and Haynes (1987) we argue that velocity histograms of wide areas are not sensitive tools for the study of the different clustering properties of galaxies of different types. The question of whether low luminosity galaxies fill the voids still remains unanswered, in our opinion, and awaits redshifts of complete samples to fainter limits than those of present surveys. At the limits sampled by our survey, the density contrast of low and high $n$ regions is substantially reduced with respect to that seen among the bright (say M> -20 ) galaxies, but still far from obliterated. The failure by several groups to detect at $21 \mathrm{~cm}$ gas clouds in voids (see Altschuler, Davis and Giovanardi 1987 and refs. therein and reports by Prof. Sancisi at this symposium) implies that objects with very low ratios of light to HI mass are rare.

These results also infringe on the concept of a universal LF of galaxies. The global LF may depend on the local density of the galaxian population : first - but possibly very mildly -, because there is morphological type segregation with $n$ and the LF of a given type $t, \Phi_{t}$, is different from that of another type (SBT), and second, because within a range of types, the shape of $\Phi_{t}$ varies 
with $n$. In a notable study of the variations of galaxy properties with local density, Dressler (1980) concluded that the LF's of high and low density regions were the same. Do Dressler's and our sample differ enough to justify the discrepancy? Dressler's low density regions correspond to the outskirts of clusters of galaxies, which in our more coarsely sampled density scale would rather fall in the high $n$ half; the population ratios E:S0:Spiral of these regions and of his "field" sample are typically 15:35:50, rather than the "canonical" 10:10:80. In contrast, our overall sample is much more spiral-rich, biased toward the opposite side in the morphology scale. Furthermore, because Dressler's sample includes relatively distant clusters (typically $10-15000 \mathrm{~km} \mathrm{~s}^{-1}$ ), his counts sample the brightest galaxies which, among the spirals, means the earlier types $(S 0 a, S a, S a b)$, whereas our sample is more heavily weighted towards the middle-to-late spirals. We postulate that these differences are sufficient to explain the discrepancies between Dressler's and our conclusions (although the discrepancy may be only a matter of quantity: a dependence on density was noted by Dressler in the LF of spirals, in the same sense albeit weaker, than that found in our data set). The bright cutoff in Dressler's sample may be also responsible for his inability to detect a difference between the LF of spirals and that of $S 0$ 's and $E$ 's, since the difference becomes more conspicuous as later spiral types are included (see SBT).

\section{SUMMARY}

The redshift survey of the Pisces-Perseus supercluster region is better than $60 \%$ complete to the limits of 15.7 in apparent magnitude and 1' in angular size. Using a subset of the data which constitutes a complete volume-limited sample, we obtain estimates of density contrasts in the bright $(\mathrm{M}<-19.5)$ galaxy counts. The mean underdensity of voids is 5 or higher, while the overdensity of large-scale density enhancements is as high as 20 . The density contrast is dependent not only on morphological type, but also on luminosity. The LF of galaxies is not a universal function : it depends on the local density of galaxies, which not only determines the population ratios of different morphological classes but also the shape of the luminosity function of individual Hubble types.

\section{REFERENCES}

Altschuler, D.R., Davis, M.M. and Giovanardi, C. 1987, Astr. Ap., 178,16

Binggeli, B. 1987, preprint

Bothun, G.D., Beers, T.C., Mould, J.R. and Huchra, J.P. 1986, Ap. J. 308,510

Davis, M. and Djorgovski 1985, Ap. J. 299,15

Dekel, A. and Rees, M.J. 1987, Nature, in press

Dressler, A. 1980, Ap. J. 236,351

Giovanelli, R. and Haynes, M.P. 1985, A. J. 90,2445

Giovanelli, R. and Haynes, M.P. 1987, in Understanding Galaxies at High Redshift, R. Kron and A. Renzini, eds., to appear.

Giovanelli, R., Haynes, M.P., Myers, S.T. and Roth, J. 1986, A. J. 92, 250

Giovanelli, R., Haynes, M.P. and Chincarini, G.L. 1986, Ap. J. 300,77

Haynes, M.P. and Giovanelli, R. 1986, Ap. J. (Letters) 396,L55

Haynes, M.P. and Giovanelli, R. 1984, A. J. 89,758

Nilson, P. 1973, Uppsala General Catalog of Galaxies, Uppsala Ann. Obs. 6 (UGC)

Oemler, A. 1987, preprint

Sandage, A., Binggeli, B. and Tamann, G.A. 1985, A. J. 90, 1759

Zwicky, F., Herzog, E., Karpowicz, M., Kowal, C.T. and Wild, P. 1961-68, Catalogue of Galaxies and of Clusters of Galaxies, 6 volumes, Pasadena: Cal. Inst. of Tech. Press (CGCG) 